\title{
Focusing concave lens using photonic crystals with magnetic materials
}

\author{
Shieh-Yueh Yang \\ Institute of Electro-optical Science and Technology, National Taiwan Normal University, Taipei 116, Taiwan
}

Chin-Yih Hong

Department of Mechanical and Automation Engineering, Da-Yeh University, Chang-hwa 515, Taiwan

Hong-Chang Yang

Department of Physics, National Taiwan University, Taipei 106, Taiwan

Received June 24, 2005; revised September 11, 2005; accepted September 30, 2005; posted October 14, 2005 (Doc. ID 62967)

The guided modes lying in the upper gap-edge band in the photonic band structure of photonic crystals have negative values of refractive index. This feature generates many interesting optical phenomena, and some spectacular photonic devices such as focusing slabs have been developed. We report the design of a photoniccrystal, planoconcave lens for focusing incident parallel light, and theoretically analyze the chromatic aberrations for TM and TE modes. In addition to dielectric photonic crystals, the chromatic aberration of a magnetic photonic-crystal planoconcave lens was investigated because the magnetic permeability may also contribute to the periodic index contrast in photonic crystals, especially at long wavelengths. A significant difference was found in the chromatic aberration for a TM mode propagating in a dielectric than in a magnetic photoniccrystal planoconcave lens. (c) 2006 Optical Society of America

OCIS codes: $220.3620,220.2560,230.3990$.

\section{INTRODUCTION}

With the valuable demonstration given by the pioneers Pendry et al..$^{1,2}$ and Smith et al. ${ }^{3}$ negative refraction has had a strong influence on optical research and has led to the development of many new types of photonic devices, such as flat focusing lenses. The original idea behind achieving negative refraction is to generate negative values of both dielectric constant and magnetic permeability at certain frequency ranges by engineering the medium architecture; for example, by using metallic split-ring resonators. ${ }^{3}$

In addition to metallic split rings, negative refraction has also been observed when a microwave is transmitted through dielectric photonic-crystal slabs. ${ }^{4-6}$ The relevant physical origin was further clarified as the guided modes that lie in the upper gap-edge band in the photonic band structure have negative refractive indices. This is so significant that more efforts have been directed toward exploring photonic crystals with desired negative refractive indices and their novel applications, like threedimensional imaging, ${ }^{4}$ evanescent-wave amplifiers, ${ }^{7}$ etc.

In this work, we report the design of a photonic-crystal, planoconcave focusing lens by utilizing negative refraction and describe the study of chromatic aberration for TM and TE modes. To do this, the photonic band structures for TM and TE modes propagating in a photonic crystal of triangularly arrayed rods are simulated. Then, the frequency-dependent refractive indices of the upper gap-edge modes are obtained, followed by calculation of the focal lengths to find the chromatic aberrations for TM and TE modes.

In addition, taking into account that negativerefraction devices using photonic crystals are very promising for microwave applications, the issue of the magnetic effect is addressed because the magnetic permeability of rods may also contribute to the periodic index contrast at long wavelengths. Hence, the chromatic aberrations of the photonic-crystal planoconcave lens incorporating magnetic rods are also studied. Here, without loss of generality, the two-dimensional photonic crystal is of triangularly arrayed, infinitely long rods surrounded by air. The ratio of the rod diameter $a$ to the rod spacing $d$ is 0.4. The rods may be either dielectric or magnetic, of dielectric constant $\epsilon_{\text {rod }}$ and magnetic permeability $\mu_{\text {rod }}$. The $\left(\epsilon_{\text {rod }}, \mu_{\text {rod }}\right)$ ratio is set to be $(15: 1)$ and (10:1.5) to investigate the magnetic effect on the chromatic aberrations for TM ( $E$ field is along the rod) and TE ( $H$ field is along the rod) modes.

\section{SIMULATION METHOD}

For magnetoactive photonic crystals, the photonic band structure can be simulated by solving the master equation $^{8,9}$

$$
\frac{1}{\mu} \nabla x \frac{1}{\varepsilon} \nabla x \frac{1}{\mu} \mathbf{B}=\left(\frac{\omega}{c}\right)^{2} \frac{1}{\mu} \mathbf{B}
$$

for the magnetic flux density $\mathbf{B}$ of electromagnetic waves propagating in the photonic crystal by the frequency- 
domain plane-wave expansion method, where $\epsilon$ is the dielectric constant, $\mu$ is the magnetic permeability, $\omega$ is the frequency of a propagation mode, and $c$ is the light speed in vacuum.

It is well known that a guided mode in a photonic crystal is a Bloch wave, which can be expressed as the product of a plane wave $\exp [i(\mathbf{k} \cdot \mathbf{r}-\omega t)]$ and a function $u_{k}(\mathbf{r})$ with the periodicity of the crystal lattice, where $\mathbf{k}$ is the wave vector of a Bloch wave and $\mathbf{r}$ is a position vector. Although the Bloch wave is not a plane wave, it is clear that the phase velocity corresponds to the propagating direction of a Bloch wave. Thus, the phase index is a key quantity connecting the incident and the outgoing directions, i.e., refraction behavior. In this work, we investigate the refraction behavior of a photonic-crystal concave lens. To do this, it is necessary to examine the phase index of guided modes in a photonic crystal. Since the calculated photonic band structure is shown as $\omega_{N}$ versus $k_{N}$, with the dimensionless frequency $\omega_{N}(=d / \lambda)$ and the dimensionless wave vector $\mathbf{k}_{N}\left(=\mathbf{k} d / 2 \pi=n_{p} d / \lambda\right)$, where $\lambda$ is the wavelength of a propagation mode, then the phase index $n_{p}$ of the propagating/guided wave can be obtained from the photonic band structure via

$$
n_{p}= \pm k_{N} / \omega_{N},
$$

for TM and TE modes propagating in a dielectric or a magnetic photonic crystal, where the + sign means positive refraction, while the - sign denotes negative refraction. With the refractive index, the focal length $f$ of a planoconcave lens is calculated via ${ }^{10}$

$$
f=R /\left(1-n_{p}\right),
$$

where $R$ is the radius of curvature of the planoconcave lens.

\section{RESULTS AND DISCUSSION}

The photonic band structure for a TM mode propagating in the photonic crystal of dielectric rods $\left(\epsilon_{\mathrm{rod}}=15, \mu_{\mathrm{rod}}=1\right)$ is plotted with dashed curves in Fig. 1(a). The first forbid-


Fig. 1. Photonic band structure of the photonic crystal made of triangularly arrayed magnetic (solid curves) or dielectric (dashed curves) rods for (a) TM, (b) TE modes. The magnetic rods have a ratio of $(10: 1.5)$ for $\left(\epsilon_{\text {rod }}, \mu_{\text {rod }}\right)$, and the dielectric rods have (15:1). The ratio of the rod radius to the rod spacing $a / 2 d$ is 0.2 .

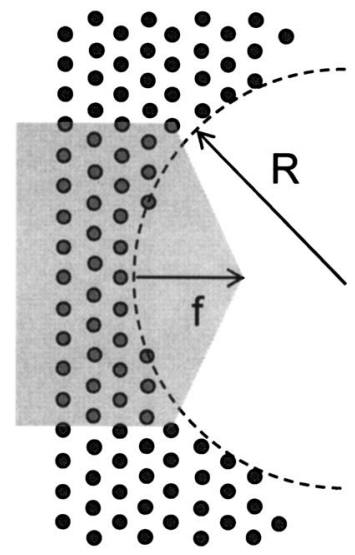

Fig. 2. Scheme of a photonic-crystal planoconcave lens. The rods are infinitely long along the direction outward from the page. The ratio of the rod radius to the rod spacing $a / 2 d$ is 0.2 . The radius of curvature of the lens is denoted by $R$. The gray shadow represents the incoming plane wave from the flat side of the lens and focused out of the concave side. The focal length is $f$.

den gap occurs at $\omega_{N}$ from 0.25 to 0.4 . For a TM mode propagating in the photonic crystal of magnetic rods $\left(\epsilon_{\text {rod }}=10, \mu_{\text {rod }}=1.5\right)$, the dispersion relations are shown by solid curves in Fig. 1(a). It is clear that the $\omega_{N}-k_{N}$ curve of a certain band shifts upward for the photonic crystal composed of magnetic rods. Thus, the forbidden frequencies become higher for a magnetic photonic crystal. The detailed discussion for the shift in the $\omega_{N}-k_{N}$ curves of the TM mode has been given in our previous paper. ${ }^{11}$ Briefly speaking, it is due to the reduction in the effective refractive index of a TM mode in the magnetic case.

Figure 1(b) shows the photonic band structures around the first forbidden band for a TE mode propagating in photonic crystals of dielectric rods (dashed curves) and magnetic rods (solid curves). In contrast to the TM mode, there is no obvious variation in the $\omega_{N}-k_{N}$ curves between the dielectric and the magnetic rods for the TE mode.

It has been demonstrated that the upper gap-edge mode, which shows a concave downward $\omega_{N}-k_{N}$ curve, possesses negative refractive indices. ${ }^{3,4}$ By utilizing the negative refraction, many novel photonic-crystal devices have been developed. In this work, we design and characterize a photonic-crystal planoconcave lens for focusing an incident parallel continuous-wave light beam. The scheme of the lens is shown in Fig. 2. The radius of curvature of the planoconcave lens is $R$. A plane-wave parallel light (denoted by the gray shadow) is normally incident on the plane side of the lens and then propagates along the $\Gamma-M$ direction in the photonic-crystal lens. When the light transmits out of the concave side of the lens, the parallel light is focused, instead of diverged, because of negative refraction. The focal length $f$ can be evaluated via Eq. (3) with known negative refractive index $n_{p}$, which is available through Eq. (2) with the aid of the photonic band structures shown in Fig. 1. For example, in the case of dielectric rods, the values of $k_{N}$ and $\omega_{N}$ in the upper gap-edge mode shown in Fig. 1(a) are 0.289 and 0.456 , respectively. Thus, the phase index $n_{p}$ is obtained as -0.634 via Eq. (2) for negative refraction.

Figures 3(a) and 3(b) plot the negative refractive indices $n_{p}$ as functions of frequency for TM and TE modes, re- 


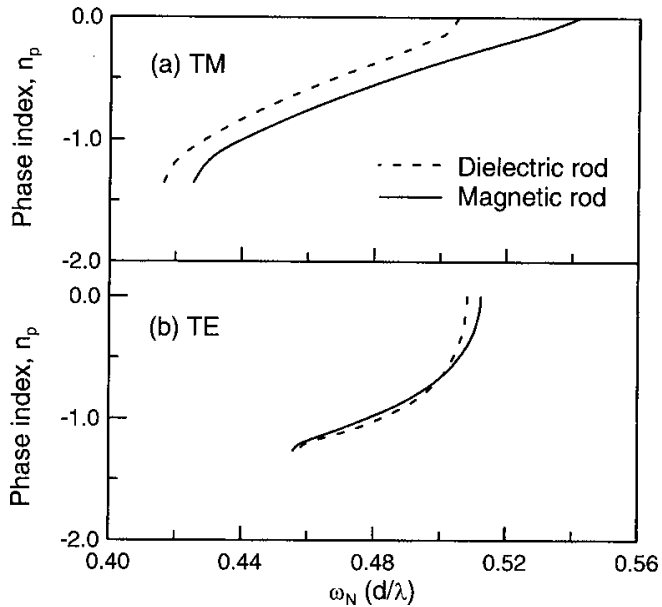

Fig. 3. Frequency-dependent phase index $n_{p}$ for (a) TM, (b) TE modes propagating along the $\Gamma-M$ direction of the photonic crystal shown in Fig. 2. The dashed/solid curves correspond to the cases where the photonic crystal is composed of dielectric/ magnetic rods with the $\left(\epsilon_{\mathrm{rod}}, \mu_{\mathrm{ro}}\right)$ ratio being (15:1) and (10:1.5).

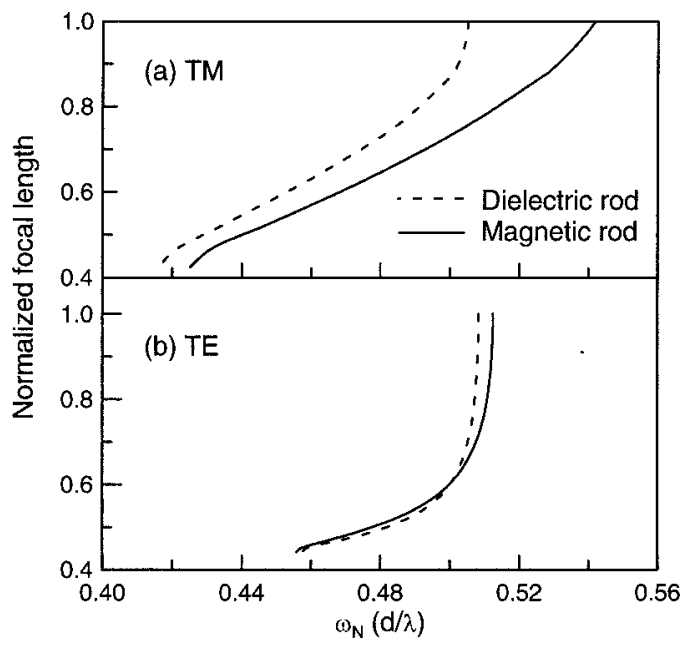

Fig. 4. Normalized focal length as a function of frequency for (a) TM, (b) TE modes propagating through the photonic-crystal planoconcave lens shown in Fig. 2. The dashed/solid curves correspond to the cases where the photonic crystal is composed of dielectric/magnetic rods with the $\left(\epsilon_{\mathrm{rod}}, \mu_{\mathrm{ro}}\right)$ ratio being $(15: 1) /(10: 1.5)$.

Table 1. Architectural Parameters for the Photonic-Crystal Planoconcave Lens ${ }^{\text {a }}$

\begin{tabular}{lcccc}
\hline & \multicolumn{4}{c}{ Parameter $(\mathrm{cm})$} \\
\cline { 2 - 5 } Mode & $d$ & $a$ & $R$ & $f$ \\
\hline $\mathrm{TM}$ & 0.48 & 0.19 & 3.31 & $\frac{2.38}{1.69}$ \\
\hline
\end{tabular}

${ }^{a}$ For the lens shown in Fig. 2 to focus the incident TM and TE modes of $1 \mathrm{~cm}$ wavelength. The $\left(\varepsilon_{\mathrm{rod}}, \mu_{\mathrm{rod}}\right)$ is $(15: 1)$, and the $a / 2 d$ is 0.2 .

spectively. The frequency range considered here corresponds to the upper edge modes of the first forbidden band along the $\Gamma-M$ direction shown in Fig. 1. It is noted that the phase index $n_{p}$ depends on the propagating direction of a guided mode. In this work, we concentrate on characterizing the upper edge modes of the first forbidden band propagating along the $\Gamma-M$ direction. The dashed curves in Figs. 3(a) and 3(b) denote the case of dielectric rods $\left(\epsilon_{\text {rod }}=15, \mu_{\text {rod }}=1\right)$; the solid curves are for magnetic rods $\left(\epsilon_{\mathrm{rod}}=10, \mu_{\mathrm{rod}}=1.5\right)$. As with the photonic band structures in Fig. 1, a clear separation of the $n_{p}-\omega_{N}$ curves between the dielectric and the magnetic rods was found for the TM mode. A larger negative value is obtained for the refractive index of the TM mode in the magnetic case. With these refractive indices at various frequencies, the frequency-dependent focal length (i.e., chromatic aberration) of the photonic-crystal planoconcave lens is analyzed via Eq. (3). The results are shown in Figs. 4(a) and 4(b) for the TM and TE mode, respectively. Here, the focal length is normalized to the radius of curvature of the lens.

For the TM mode focused by a dielectric photoniccrystal lens, the normalized focal length increases from 0.42 to 1 as the frequency varies from 0.416 to 0.505 , as shown by the dashed curve in Fig. 4(a). This implies that the incoming TM modes with various frequencies are focused at different positions and reveals the high chromatic aberration of the photonic-crystal planoconcave lens. This high chromatic aberration is attributed to the highly dispersive refractive index shown in Fig. 3(a). A trend similar to the dielectric case is observed for the chromatic aberration of the TM mode focused by a magnetic photonic-crystal lens, except a shift is seen to lower values for the normalized focal length. The photoniccrystal planoconcave lens also exhibits a high chromatic aberration for the TE mode, as shown in Fig. 4(b). In either dielectric or magnetic case, the normalized focal length is 0.42 at the frequency of $\approx 0.425$ and rises monotonically to 1 when the frequency reaches 0.541 .

Here we have a clear example how to design a realistic dielectric, photonic-crystal, planoconcave lens to focus a TM mode of a certain wavelength, say $1 \mathrm{~cm}$. According to the photonic band structure in Fig. 1(a), the rod spacing $d$ is selected as $0.48 \mathrm{~cm}$ to make the dimensionless frequency $\omega_{N}=0.48$. Thus, this incoming TM-mode plane wave is one of the upper gap-edge modes and exhibits a negative refractive index of -0.4 , as obtained from Fig. 3 (a). Since the $a / 2 d$ is 0.2 for the investigated photonic crystal, the diameter of the rod $a$ is $0.19 \mathrm{~cm}$. From the dashed curve in Fig. 4(a), the normalized focal length $f / R$ at the frequency of 0.48 is 0.72 . Hence, the focal length of the photonic-crystal planoconcave lens is $0.72 R$ for the TM-mode plane wave. For the lens with the architecture shown in Fig. 2, with $d=0.48 \mathrm{~cm}$ and $R=3.31 \mathrm{~cm}$, the focal length $f$ of the TM-mode plane wave is $2.38 \mathrm{~cm}$ via $f / R=0.72$ in the current case. For an incoming TE-mode plane wave, the focal length becomes $1.69 \mathrm{~cm}$. All the parameters are listed in Table 1. One can follow the same philosophy to design a photonic-crystal planoconcave lens for practical applications.

\section{CONCLUSION}

By utilizing negative refraction, a parallel incident light beam that correspondingly lies in the upper gap-edge band of a photonic crystal can be focused with a photoniccrystal planoconcave lens. A high chromatic aberration is found for the focal length of the lens, which results from the high dispersion in the frequency-dependent refractive 
index of the upper gap-edge band. In addition, the TM mode shows significantly different chromatic aberration when focused with a dielectric or a magnetic photoniccrystal planoconcave lens.

\section{ACKNOWLEDGEMENTS}

This work is supported by the National Science Council of Taiwan under grant NSC93-2112-M-003-007 and 942112-M-003-004.

Corresponding author H.-C. Yang's e-mail address is hcyang@phys.ntu.edu.tw.

\section{REFERENCES}

1. B. Pendry, A. J. Holden, W. J. Stewart, and I. Youngs, "Extremely low frequency plasmons in metallic mesostructures," Phys. Rev. Lett. 76, 4773-4776 (1996).

2. J. B. Pendry, A. J. Holden, D. J. Robbins, and W. J. Stewart, "Magnetism from conductors and enhanced nonlinear phenomena," IEEE Trans. Microwave Theory Tech. 47, 2075-2084 (1999).

3. D. R. Smith, W. J. Padilla, D. C. Vier, S. C. Nemat-Nasser, and S. Schultz, "Composite medium with simultaneously negative permeability and permittivity," Phys. Rev. Lett. 84, 4184-4186 (2000).

4. M. Notomi, "Negative refraction in photonic crystals," Opt. Quantum Electron. 34, 133-143 (2002).

5. C. Luo, S. G. Johnson, and J. D. Joannopoulos, "All-angle negative refraction in a three-dimensionally periodic photonic crystal," Appl. Phys. Lett. 81, 2352-2354 (2002).

6. K. Guven, K. Aydin, K. B. Alici, C. M. Soukoulis, and E. Ozbay, "Spectral negative refraction and focusing analysis of a two-dimensional left-handed photonic crystal lens," Phys. Rev. B 70, 205125-205129 (2004).

7. A. Husakou and J. Herrmann, "Superfocusing of light below the diffraction limit by photonic crystals with negative refraction," Opt. Express 12, 6491-6497 (2004).

8. I. Drikis, S. Y. Yang, H. E. Horng, C.-Y. Hong, and H. C. Yang, "Modified frequency-domain method for simulating the electromagnetics in periodic magnetoactive systems," J. Appl. Phys. 95, 5876-5881 (2004).

9. S. Y. Yang, C.-Y. Hong, I. Drikis, H. E. Horng, and H. C. Yang, "Photonic characterizations of triangular-arrayed rods with both dielectric and magnetic permeability functions," J. Opt. Soc. Am. B 21, 413-418 (2004).

10. D. Halliday, R. Resnick, and J. Walker, Fundamentals of Physics, 6th ed. (Wiley, 2001), Chap. 35, pp. 841-846.

11. S.-Y. Yang and C. T. Chang, "Birefringence prism made of photonic crystals using magnetic material," submitted to J. Opt. Soc. Am. B. 